\title{
Mycobacterium tuberculosis DNA facilitates selective autophagy within infected macrophages
}

Selective autophagy is a housekeeping energy-generating cytosolic process through which organelles and protein aggregates are tagged within a double-membrane autophagosome and delivered to the lysosome for proteolysis releasing amino acids and generating ATP. The molecular mechanisms underlying protein selection for degradation and ubiquitin complex formation within the cytosol are yet to be elucidated.

The authors describe a novel molecular process through a series of experiments using bone marrow-derived macrophages infected with wild-type Mycobacterium tuberculosis (MTB). They demonstrate that MTB possesses a pore-forming enzyme system ESX-1 that exposes the MTB-containing vacuole to the cytosol. The exposure of mycobacterial DNA attracts ubiquitin and ubiquitin-binding cytosolic receptors, p62 and NADP52, through activation of the DNA-sensing STING pathway. This recruits the protein LC3 that forms puncta within the autophagosome marking it for fusion with the lysosome. They were able to demonstrate that this process is driven by MTB DNA, that bacteria targeted are killed within phagolysosomes and that this is essential for in vivo control of MTB infection-demonstrated by a fall in the number of colony-forming units within $6 \mathrm{~h}$ and $24 \mathrm{~h}$ post-infection.

The work sheds new light on the processes through which MTB is cleared within macrophages, adding to the growing interest over the last decade on the cellular mechanisms underlying autophagy. It paves the way for the molecular pathways underlying MTB resistance to be elucidated and in turn identify appropriate targets for therapeutic intervention.

- Watson JO, Manzanillo PS, Cox JS. Extracellular M. tuberculosis DNA targets bacteria for autophagy by activating the host DNA-sensing pathway. Cell 2012;150:803-15.

\section{Rawya Ahmed}

Correspondence to Rawya Ahmed; rkia@doctors.org.uk

Competing interests None.

Provenance and peer review Not commissioned; internally peer reviewed.

To cite Ahmed R. Thorax 2013;68:895.

Published Online First 27 October 2012

Thorax 2013;68:895. doi:10.1136/thoraxjnl-2012-202844 